\section{Erlenmeyer, Emil}

T. Arndt

Bioscientia Institut für Medizinische Diagnostik $\mathrm{GmbH}$, Ingelheim, Deutschland
Lebensdaten Deutscher Chemiker, geb. am 28. Juni 1825 in Taunusstein, gest. am 22. Januar 1909 in Aschaffenburg.

Verdienste Professor der Chemie in Heidelberg und München. Klärte die Strukturen von Naphthalin, Azo-, Hydrazound Azoxykörpern auf. Herausgeber von „Liebigs Annalen“. Erfinder des Erlenmeyer-Kolbens. 